\title{
Efficacy of ampicillin against methicillin-resistant Staphylococcus aureus restored through synergy with branched poly(ethylenimine)
}

\author{
Melissa A Foxley ${ }^{1}$, Anthony W Friedline ${ }^{1}$, Jessica M Jensen ${ }^{1}$, Susan L Nimmo ${ }^{1}$, Erin M Scull ${ }^{1}$, Jarrod B King ${ }^{1}$, \\ Stoffel Strange ${ }^{1}$, Min T Xiao' ${ }^{1}$, Benjamin E Smith ${ }^{2}$, Kieth J Thomas III ${ }^{1}$, Daniel T Glatzhofer ${ }^{1}$, \\ Robert H Cichewicz ${ }^{1}$ and Charles V Rice ${ }^{1}$
}

$\beta$-Lactam antibiotics kill Staphylococcus aureus bacteria by inhibiting the function of cell wall penicillin-binding proteins (PBPs) 1 and 3. However, $\beta$-lactams are ineffective against PBP2a, used by methicillin-resistant $S$. aureus (MRSA) to perform essential cell wall crosslinking functions. PBP2a requires teichoic acid to properly locate and orient the enzyme, and thus MRSA is susceptible to antibiotics that prevent teichoic acid synthesis in the bacterial cytoplasm. As an alternative, we have used branched poly(ethylenimine), BPEI, to target teichoic acid in the bacterial cell wall. The result is restoration of MRSA susceptibility to the $\beta$-lactam antibiotic ampicillin with a MIC of $1 \mu \mathrm{g} \mathrm{ml}^{-1}$, superior to that of vancomycin $\left(\mathrm{MIC}=3.7 \mu \mathrm{g} \mathrm{ml}^{-1}\right.$ ). A checkerboard assay shows synergy of BPEI and ampicillin. NMR data show that BPEI alters the teichoic acid chemical environment. Laser scanning confocal microscopy images show BPEI residing on the bacterial cell wall, where teichoic acids and PBPs are located.

The Journal of Antibiotics (2016) 69, 871-878; doi:10.1038/ja.2016.44; published online 18 May 2016

\section{INTRODUCTION}

Methicillin-resistant Staphylococcus aureus (MRSA) is a current and growing risk to human health. It causes serious infections that show remarkable resistance to antibiotic treatment. ${ }^{1}$ Originally acquired exclusively in health-care settings, MRSA is now regularly found outside the health-care environment. ${ }^{2}$ Drug resistance hinders efforts to develop safe clinical treatments for MRSA infections. ${ }^{3}$ Fortunately, progress has been made toward developing new antibiotics such as oxadiazoles, ${ }^{4}$ tedizolid ${ }^{5}$ and teixobactin. ${ }^{6}$ The timing coincides with a critical period in antibiotic research and development as MRSA is developing resistance to drugs of last resort. ${ }^{1,7,8}$ Therapeutic approaches to overcome resistance factors include efflux pump inhibitors that increase the intracellular concentration of antibiotics. ${ }^{9}$ Bacteria can also use $\beta$-lactamase enzymes that degrade the antibiotics ${ }^{10}$ and thus treatment requires $\beta$-lactamase inhibitors. ${ }^{11}$

The cell envelope of Gram-positive bacteria is composed of a membrane, peptidoglycan and teichoic acids (Figure 1a). ${ }^{6}$ Methicillin, a $\beta$-lactam antibiotic, occupies the active site of penicillin-binding proteins (PBP) 1 and 3 to prevent the enzymatic cell wall synthesis function (Figure 1b). MRSA performs cell wall synthesis using PBP2a (Figure 1c), a transpeptidase enzyme with very low affinity for $\beta$-lactams. Hartman and Tomasz ${ }^{12}$ recognized and identified PBP2a in MRSA. The $\beta$-lactam/transpeptidase complex is stable; however, resistance arises because the rate of complex formation is much slower than the $S$. aureus cell division time. Thus, it is nearly impossible for the complex to form in vivo. ${ }^{13}$ Fuda et al. also presented a structure of PBP2a with no realistic access to the active site, suggesting there had to be a conformational change that took place as a result of binding non-crosslinked peptidoglycan at a location other than the active site, setting the groundwork for future investigations of allosteric regulation. Nevertheless, the cell wall remains an especially rich antimicrobial target, containing many opportunities for disruption, such as excess peptidoglycan, ${ }^{14}$ teichoic acids ${ }^{15,16}$ and novel proteins. ${ }^{17}$ Although these targets have shown promise, side effects and slow progress toward clinical usage have hindered efforts to reduce the rate of MRSA infection and mortality. ${ }^{18}$ Although it is possible to stop teichoic acid expression in the cytoplasm, thereby disabling the function of $\mathrm{PBP} 2 \mathrm{a},{ }^{15}$ the quantity of drug required for activity prevent development into a clinical MRSA treatment. ${ }^{18}$

The continued spread of resistance could be stemmed by resensitizing resistant strains of $S$. aureus to currently ineffective antibiotics, such as the $\beta$-lactam ampicillin. This approach can be viable by inhibiting the expression and/or functionality of proteins that contribute to resistance, such as PBP2a. PBPs are indispensable for cell growth as they create essential crosslinks between adjacent peptidoglycan segments. Targeting PBP2a with inhibitors has been

${ }^{1}$ Department of Chemistry and Biochemistry, Stephenson Life Sciences Research Center, University of Oklahoma, Norman, OK, USA and ${ }^{2}$ Samuel Roberts Noble Microscopy Laboratory, University of Oklahoma, Norman, OK, USA

Correspondence: Dr CV Rice, Department of Chemistry and Biochemistry, Stephenson Life Sciences Research Center, University of Oklahoma, 101 Stephenson Parkway, Norman, OK 73019, USA.

E-mail: rice@ou.edu

Received 11 November 2015; revised 22 March 2016; accepted 29 March 2016; published online 18 May 2016 
a Representation of Cell Wall Components for Gram positive bacteria

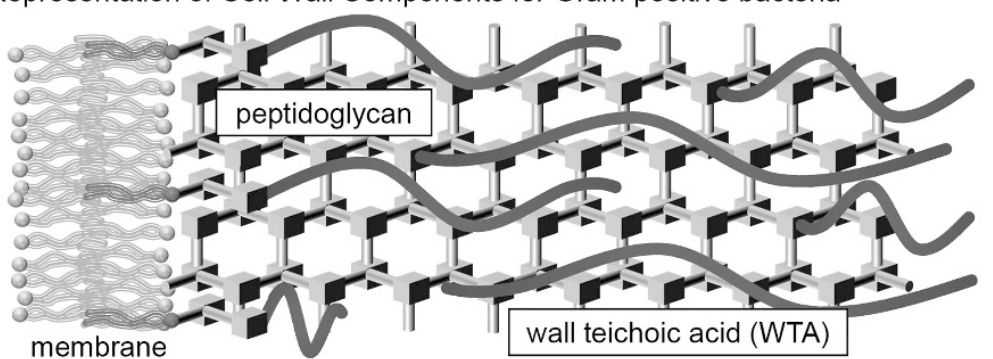

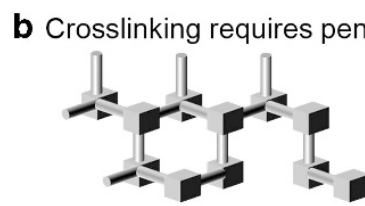

c MRSA uses PBP2a

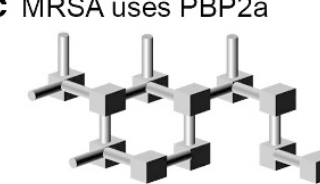

1
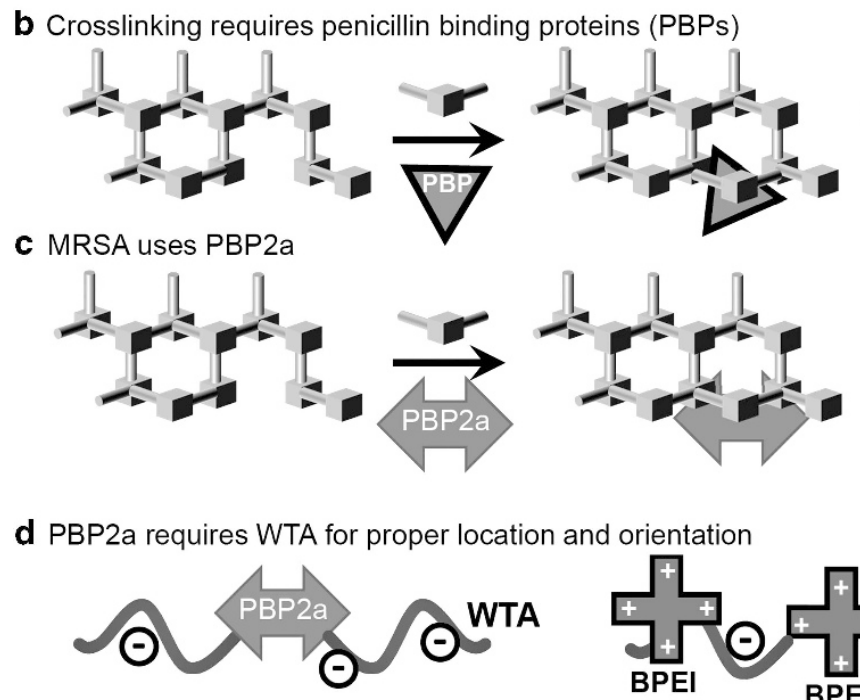

Figure 1 Schematic $^{6}$ of Gram-positive cell wall components (a) and crosslinking of peptidoglycan using PBPs 1 and 3, which can be inhibited by $\beta$-lactams (b) and PBP2a, which cannot (c). PBP2a requires WTA to be properly localized; BPEI can bind to WTA to prevent PBP2a from functioning properly (d). BPEI, branched poly(ethylenimine); PBPs, penicillin-binding proteins; WTA, wall teichoic acid. A full color version of this figure is available at The Journal of Antibiotics journal online.

shown to resensitize resistant strains to methicillin. ${ }^{19}$ In contrast, branched poly(ethylenimine), BPEI, may indirectly disable PBP2a. Our work shows that BPEI, administered in concert with ampicillin, resensitizes MRSA to ampicillin. Laser scanning confocal microscopy (LSCM) images show that BPEI binds to the cell wall, where it can interrupt the function of teichoic acids, inactivate PBP2a and restore $\beta$-lactam antibiotic activity. NMR spectroscopy data demonstrate that BPEI binds to teichoic acids, likely through ionic attraction between the cationic polymer and the anionic teichoic acid. Ampicillin activity against MRSA was restored by low-MW BPEI. Checkerboard assays were used to measure the fractional inhibitory concentration (FIC) index and identify synergy between $1-8 \mu \mathrm{g} \mathrm{ml}^{-1}$ ampicillin and $16 \mu \mathrm{g} \mathrm{ml}^{-1}$ low-MW BPEI; or $8 \mu \mathrm{g} \mathrm{ml}^{-1}$ ampicillin and $8 \mu \mathrm{g} \mathrm{ml}^{-1}$ low-MW BPEI. However, BPEI does not increase the efficacy of vancomycin or novobiocin. Thus, BPEI's synergistic properties arise by indirectly disabling PBP2a, rendering MRSA susceptible to ampicillin that disables PBP1 and PBP3.

\section{RESULTS AND DISCUSSION}

Although there are numerous MRSA strains, S. aureus subsp. aureus ATCC 700787 (ATCC, Manassas, VA, USA) was isolated in Port Chester, NY, USA from blood culture, exhibits intermediate resistance to vancomycin ${ }^{20}$ and also expresses mecA to produce PBP2a. ${ }^{21}$ The ability of BPEI to restore ampicillin effectiveness against MRSA is shown in Table 1 . The growth of MRSA in vitro after $20 \mathrm{~h}$ of incubation was inhibited by ampicillin when the antibiotic was coadministered with low-MW BPEI. MRSA shows resistance toward ampicillin, with a MIC of $32 \mu \mathrm{g} \mathrm{ml}^{-1}$, but the presence of low-MW BPEI $\left(16 \mu \mathrm{g} \mathrm{ml}^{-1}\right)$ rendered MRSA susceptible to ampicillin at a $32 \times$ lower dose $\left(\mathrm{MIC}=1 \mu \mathrm{g} \mathrm{ml}^{-1}\right)$. At a reduced BPEI concentration of $8 \mu \mathrm{g} \mathrm{ml}^{-1}$, the ampicillin MIC was still decreased, but only to $8 \mu \mathrm{g} \mathrm{ml}^{-1}$. In the absence of ampicillin, BPEI itself inhibited growth of MRSA at a concentration of $64 \mu \mathrm{g} \mathrm{ml}^{-1}$. With these values, it is possible to calculate the FIC index ${ }^{22,23}$ for each combination. When the FIC is $\leqslant 0.5$, ampicillin and low-MW BPEI stop MRSA growth through synergistic effects. Synergy occurred between $8 \mu \mathrm{g} \mathrm{ml}^{-1}$ ampicillin and $8 \mu \mathrm{g} \mathrm{ml}^{-1}$ low-MW BPEI (FIC $=0.375$ ), as well as with combinations of $16 \mu \mathrm{g} \mathrm{ml}^{-1}$ low-MW BPEI with $1,2,4$ and $8 \mu \mathrm{g} \mathrm{ml}^{-1}$ ampicillin ( $\mathrm{FIC}=0.281,0.313,0.275$ and 0.500 , respectively). Although MRSA does not grow in the presence of $0.5 \mu \mathrm{g} \mathrm{ml}^{-1}$ ampicillin and $32 \mu \mathrm{g} \mathrm{ml}^{-1}$ low-MW BPEI, the FIC index is 0.516 .

BPEI's potentiation of ampicillin to inhibit MRSA growth suggests that similar effects may be seen with other antibiotics. However, low-MW BPEI did not benefit vancomycin or novobiocin against MRSA (Supplementary Figures 1 and 2, respectively). These data suggest that BPEI potentiation depends on the class of antibiotic used. Novobiocin, an aminocoumarin, works by inhibiting DNA gyrase. ${ }^{24}$ Vancomycin, a glycopeptide, inhibits peptidoglycan crosslinking by binding to the peptidoglycan stems. ${ }^{25}$ In contrast, ampicillin occupies the active sites of transpeptidase proteins that create the crosslinks between peptide stems. 
Table 1 MRSA growth Inhibition assay in the presence of ampicillin and low-MW BPEI

\begin{tabular}{|c|c|c|c|c|c|c|c|c|c|c|c|}
\hline $\begin{array}{c}\text { BPEI } \\
\text { Conc. } \\
\left(\mu \mathrm{g} \mathrm{m}^{-1}\right)\end{array}$ & \multicolumn{10}{|c|}{ Ampicillin Concentration $\left(\mu \mathrm{g} \mathrm{m}^{-1}\right)$} \\
\hline 0 & 0.4887 & 0.4361 & 0.4282 & 0.4342 & 0.4289 & 0.4294 & 0.4375 & 0.4344 & 0.3287 & 0.0003 & 0.0014 \\
\hline 1 & 0.5088 & 0.4124 & 0.4289 & 0.4310 & 0.4324 & 0.4283 & 0.4097 & 0.3943 & 0.0661 & -0.0024 & -0.0037 \\
\hline 2 & 0.4937 & 0.4388 & 0.4374 & 0.4510 & 0.4372 & 0.4540 & 0.3814 & 0.2333 & 0.0062 & -0.0020 & -0.0021 \\
\hline 4 & 0.5054 & 0.4217 & 0.4693 & 0.4289 & 0.4373 & 0.4115 & 0.2587 & 0.1954 & -0.0006 & -0.0033 & -0.0018 \\
\hline 8 & 0.4835 & 0.4423 & 0.4449 & 0.4610 & 0.2826 & 0.2411 & 0.1070 & $\mathbf{- 0 . 0 0 1 8}$ & -0.0011 & -0.0025 & -0.0002 \\
\hline 16 & 0.4066 & 0.3178 & 0.2238 & 0.2931 & $\mathbf{0 . 0 4 1 6}$ & $\mathbf{- 0 . 0 0 5 3}$ & $\mathbf{- 0 . 0 0 1 7}$ & $\mathbf{- 0 . 0 0 1 2}$ & 0.0003 & -0.0006 & -0.0007 \\
\hline 32 & 0.1860 & 0.0997 & 0.1531 & -0.0007 & 0.0798 & 0.0006 & -0.0022 & 0.0023 & 0.0009 & 0.0001 & 0.0002 \\
\hline 64 & 0.0001 & 0.0012 & 0.0005 & -0.0003 & -0.0001 & 0.0003 & 0.0006 & -0.0018 & 0.0175 & 0.0003 & 0.0011 \\
\hline
\end{tabular}

Abbreviations: BPEI, branched poly(ethylenimine); MRSA, methicillin-resistant Staphylococcus aureus.

MRSA cells (ATCC 700787) were used to inoculate growth media containing ampicillin and low-MW BPEI at $37{ }^{\circ} \mathrm{C}$ for $20 \mathrm{~h}$. Table entries are the OD of the growth media measured at a wavelength of $600 \mathrm{~nm}$. Each entry is the average of duplicate measurements. The bold line separates combinations that allowed MRSA growth (OD 600 values $>0.05)$ from those combinations that prevented MRSA growth. The ampicillin MIC is $32 \mu \mathrm{g} \mathrm{ml}^{-1}$ demonstrating antibiotic resistance without the presence of BPEI. Values highlighted in bold text were determined to arise from synergy between ampicillin and BPEI.

The trend of lower ampicillin MICs with higher BPEI concentrations suggests therapeutic treatment of MRSA using Food and Drug Administration (FDA)-approved $\beta$-lactam antibiotics may be viable. To be clinically viable, BPEI must be safe for human use. Low-MW BPEI does not cause hemolysis ${ }^{26}$ and is non-toxic, ${ }^{27}$ as cytotoxicity and renal failure are more prevalent with high-MW polymers. ${ }^{26,28,29}$ Both linear and branched forms, LPEI and BPEI, are used as non-viral transfection agents for gene therapy. ${ }^{30-32}$ In this application, LPEI has undergone clinical trial testing and is awaiting final FDA approval for gene delivery to patients (http://www.polyplus-transfection.com/ products/cgmp-grade-in-vivo-jetpei/). ${ }^{33}$ These studies also report that the cytotoxicity of PEI increases as its MW increases ${ }^{31,34}$ and that most branched PEIs are non-toxic $<25 \mathrm{kDa}^{35}$ To test the cytotoxicity of BPEI used in these experiments (MW $\sim 0.5 \mathrm{kDa}$ ), mouse fibroblasts $(\mathrm{NIH} / 3 \mathrm{~T} 3$ cells) were incubated over 3 days in the presence of varying concentrations of BPEI (Supplementary Information; Supplementary Figure 3). Viability was determined using the MTT assay, following the protocol of Hansen et al. ${ }^{36}$ At $2.65 \mu \mathrm{g} \mathrm{ml}^{-1}$, only $1.5 \%$ of the fibroblasts became nonviable over the 3 -day incubation period compared against the control sample. This indicates negligible cytotoxicity from low-MW BPEI. From the checkerboard assay (Table 1), synergy occurs with $16 \mu \mathrm{g} \mathrm{ml}^{-1}$ low-MW BPEI that causes $\sim 10 \%$ reduction in fibroblast viability (Supplementary Figure 3). At $26.5 \mu \mathrm{g} \mathrm{ml}^{-1}$ low-MW BPEI, only a $16.3 \%$ reduction in viability compared with the control sample was observed. Thus, BPEI concentrations that induce synergy are also associated with low cytotoxicity, providing guarded optimism in conducting in vivo testing. Low-MW BPEI is non-toxic, ${ }^{27}$ whereas high-MW BPEI penetrates mammalian cells leading to cytotoxicity and renal failure. ${ }^{26,28,29}$ Low-MW BPEI, up to $1000 \mu \mathrm{g} \mathrm{ml}^{-1}$, does not affect the membrane of human cells as judged by the lack of hemolysis in red blood cells and the lack of lactate hydrogenase leakage from HEp-2 cells. $^{26}$ The BPEI concentration for this toxicity testing is $\sim 25$ times larger than the highest amount, $64 \mu \mathrm{g} \mathrm{ml}^{-1}$, used for antibiotic potentiation.

Antibiotic potentiation by BPEI does not extend to Gram-negative Escherichia coli (ATCC 11775). Supplementary Information show results of growing $E$. coli in the presence of ampicillin alone and with a fixed BPEI concentration $\left(2.65 \mu \mathrm{g} \mathrm{ml}^{-1}\right.$, Supplementary Figure 4), whereas Supplementary Figure 8 shows the result of testing BPEI with a fixed ampicillin concentration $\left(0.37 \mu \mathrm{g} \mathrm{ml}^{-1}\right)$. In the first data set, the observed ampicillin MIC was identical at $3.7 \mu \mathrm{g} \mathrm{ml}^{-1}$ with or without BPEI addition. In the second data set, BPEI did not show inhibitory effects on growth of E. coli up to $53 \mu \mathrm{g} \mathrm{ml}^{-1}$, either by itself or with $0.37 \mu \mathrm{g} \mathrm{ml}^{-1}$ ampicillin. This is in contrast to the MRSA data, in which the ampicillin MIC decreased by a factor of 32 when the BPEI concentration was $16 \mu \mathrm{g} \mathrm{ml}^{-1}$. In addition, our data differs from previously reported results showing PEI-induced antibiotic synergy against Gram-negative bacteria, including E. coli and Pseudomonas aeruginosa. ${ }^{37,38}$ This could be due to differences in branching or MW between the polymers tested. Our tests use a low-MW BPEI, whereas the prior reports use a high-MW LPEI.

The data in Table 1 suggests that BPEI may interact with PBP2a. This would prevent the enzyme from functioning properly, although allowing the $\beta$-lactam to disable PBP1 and PBP3. If true, BPEI's interaction with MRSA should be confined to the cell wall. By conjugating BPEI to a fluorescent marker, Alexa Fluor 488 (Thermo Fisher Scientific, Waltham, MA, USA), we were able to visualize BPEI localization in bacterial cultures using LSCM. Individual transverse optical sections clearly show BPEI interaction with the MRSA cell wall region (Figure 2a). Using DAPI, a DNA-binding fluorescent dye, as a marker for the cytoplasm within the cells (Figure 2b) the merged image (Figure 2c) confirms that BPEI was not detected within the cytoplasm, verifying that BPEI does not traverse the lipid bilayer membrane. Similar optical sections of E. coli cells treated with BPEI conjugated Alexa Fluor 488 revealed minimal fluorescence intensity within the cell envelope, indicating a weaker interaction between the $E$. coli cell envelope and BPEI (Figure $2 \mathrm{~d}-\mathrm{f}$ ). This may explain the absence of antibiotic potentiation against $E$. coli in our present study. Additional LSCM images of the samples are provided as Supplementary Information (Supplementary Figure 8).

The microscopy data, showing BPEI located in the cell wall region and not the cytoplasm, suggest that the observed antibiotic potentiation against MRSA is caused by an interaction of BPEI, with some component of the bacterial cell wall. One major component of the Gram-positive cell wall is teichoic acid, a phosphodiester polymer whose anionic phosphate groups have been shown to interact strongly with metal cations. ${ }^{39-41} \mathrm{BPEI}$, with its polycationic properties, has the potential for very strong electrostatic interactions with the polyanionic WTA molecules. This interaction would involve the primary amines of BPEI and the phosphate groups of WTA (Figure 3). This interaction can be observed using NMR studies of mixed BPEI-teichoic acid solutions when compared with NMR spectra of teichoic acid alone.

The one-dimensional ${ }^{31} \mathrm{P}$ spectra (Figure $4 \mathrm{a}$ and $\mathrm{b}$ ) show significant changes after mixing WTA with low-MW BPEI. WTA is a phosphodiester polymer with heterogeneous arrangement of 

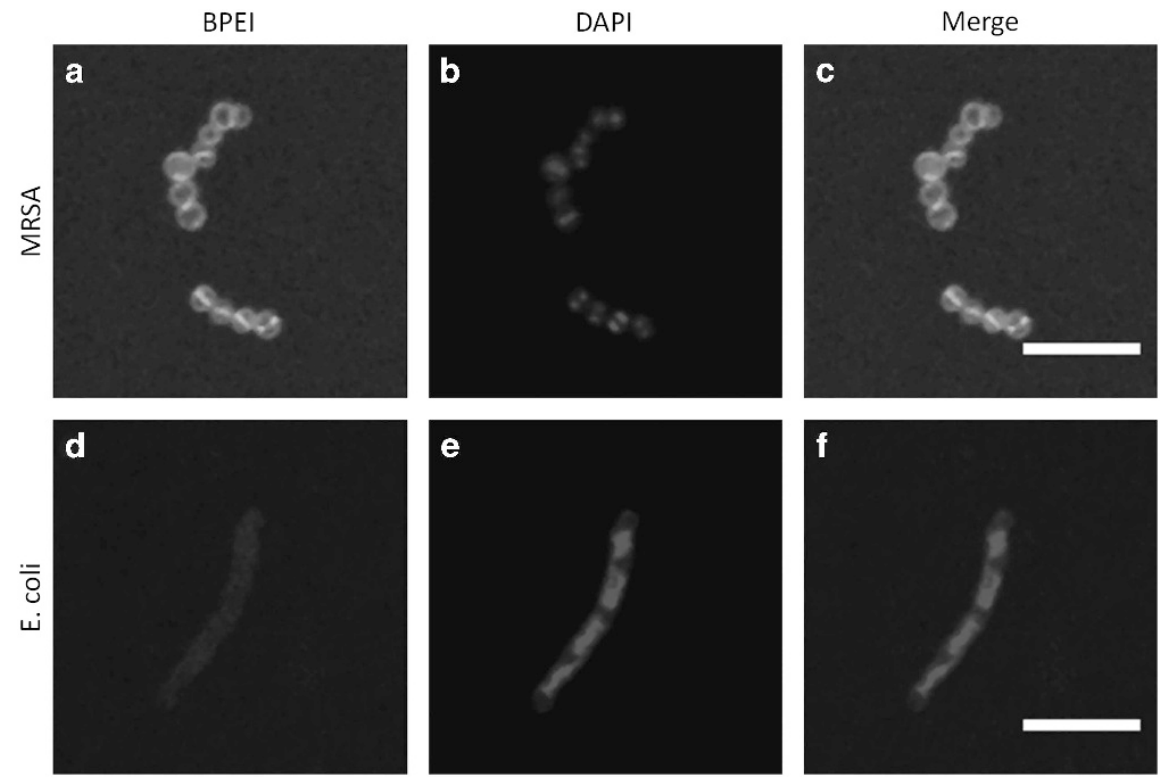

Figure 2 Optical sections of BPEI binding to MRSA and E. coli. Paraformaldehyde (PFA)-fixed MRSA, stained with BPEI-Alexa Fluor 488 (a) and DAPI (b), is imaged by LSCM. The merged image (c) shows BPEI binding to the cell surface, but not within the cytoplasm. In contrast, PFA-fixed E. coli stained with BPEI-Alexa Fluor 488 (d) and DAPI (e), and the merged image (f), shows a relatively low affinity between BPEI and $E$. coli. Scale bar $=5 \mu \mathrm{m}$. BPEI, branched poly(ethylenimine); MRSA, methicillin-resistant Staphylococcus aureus; LSCM, laser scanning confocal microscopy. A full color version of this figure is available at The Journal of Antibiotics journal online.

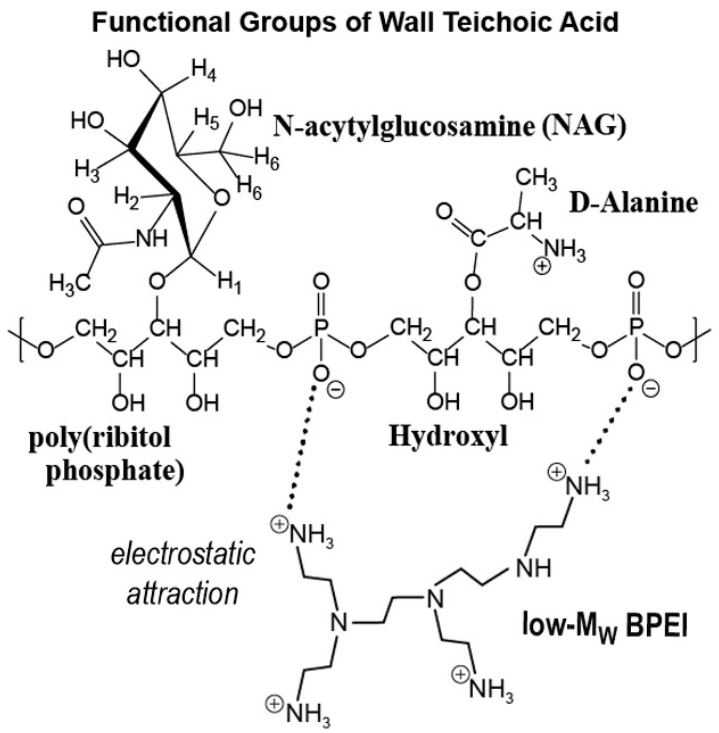

Figure 3 Chemical structure of the interaction between BPEI and the functional groups of wall teichoic acid. The cationic polymer is attracted to the anionic WTA. The result is a heterogeneous macromolecular complex that likely has increased steric bulk compared with WTA alone. These factors may prevent WTA from arranging PBP2a in its proper orientation required for crosslinking of peptidoglycan. BPEI, branched poly(ethylenimine); PBP, penicillin-binding protein.

$\mathrm{N}$-acetylglucosamine, D-alanine and hydroxyl groups. This creates variations in conformation of the poly(ribitol) backbone and differences in the phosphate conformations that generate distinct ${ }^{31} \mathrm{P}$ NMR peaks. In the presence of low-MW BPEI, the ${ }^{31} \mathrm{P}$ NMR peak at 1.3 p.p.m. is very intense, demonstrating that a large fraction of the phosphates have similar conformations. However, signals near 4 p.p.m. are produced by phosphates in a deshielded environment.
The downfield shift arises from a loss of electron density around the phosphorous nucleus, an effect that could be caused by a hydrogen bond between the phosphate oxygen and a BPEI amine group. The addition of BPEI also increases the intensity of cross peaks in the ${ }^{1} \mathrm{H}\left\{{ }^{31} \mathrm{P}\right\}$ HMBC NMR data (Figure $4 \mathrm{c}$ and $\mathrm{d}$ ). This experiment relies on strong through-bond coupling between the ${ }^{1} \mathrm{H}$ and ${ }^{31} \mathrm{P}$ nuclei. For flexible molecules, internal motion and dynamics causes relaxation of NMR signals ${ }^{42,43}$ and thus the ${ }^{1} \mathrm{H}\left\{{ }^{31} \mathrm{P}\right\}$ HMBC signals are difficult to observe. When molecular motion is restricted, the signals are stronger.

Phosphate:amine binding from the WTA:BPEI interactions likely occurs through electrostatic attraction between the numerous cationic primary amines of BPEI and anionic phosphate groups of WTA (Figure 3). If this assumption is correct linear PEI, with only the two primary amines at its terminal ends, should not affect ampicillin's MIC values. The $0.6 \mathrm{kDa}$ form of LPEI (similar in mass to the $0.5 \mathrm{kDa}$ BPEI) does not inhibit MRSA growth (Supplementary Information; Supplementary Figure 7) until its concentration is very high $\left(54 \mu \mathrm{g} \mathrm{ml}^{-1}\right)$. Low-MW quaternary ammonium compounds have recently been shown to overcome resistance if the number of cationic sites is increased. ${ }^{44}$ Therefore, the optimal cationic amine polymer should have a high number of primary amines, although minimizing cytotoxicity with a low MW.

Because low-MW BPEI binds to WTA, the cationic polymer has the ability to change WTA properties by altering molecular structure and/or creating steric bulk from the branched polymer (Figure 1d). This would change, or prevent, the interaction of WTA with PBP2a and thus disable the enzyme. The same effect can be created through WTA-deficient strains of MRSA, which are resensitized to amoxicillin, ampicillin, methicillin, nafcillin and ceftizoxime. ${ }^{15}$ An inhibitor of WTA synthesis, tunicamycin, resensitizes MRSA to $\beta$-lactams such as methicillin, oxacillin, cefotaxime and several others. ${ }^{45}$ Inhibition of another regulatory gene, $\operatorname{tar} G$, also resensitizes MRSA strains to traditional $\beta$-lactams ${ }^{46-48}$ like imipenem. ${ }^{16}$ Thus WTA, although not essential to viability, ${ }^{46-48}$ is involved in $\beta$-lactam resistance. ${ }^{49}$ WTA 
helps to optimally localize PBP2a, and WTA-deficient mutants show a decreased functionality of the protein. ${ }^{15}$ It additionally localizes PBP4, which is essential for the highly crosslinked peptidoglycan exhibited
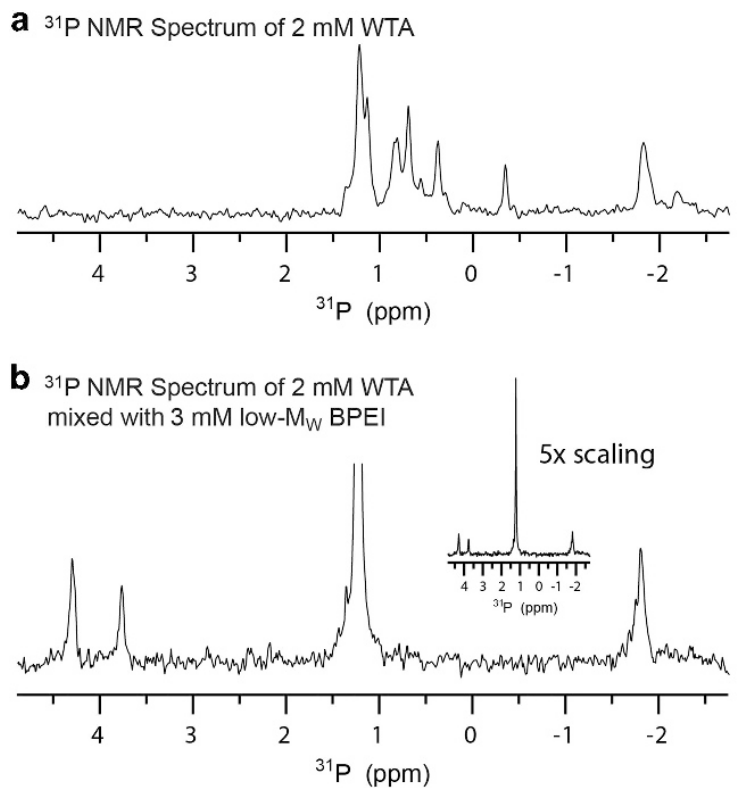

C ${ }_{1 \mathrm{H} / 31 \mathrm{P}} \mathrm{HMBC}$ NMR Spectrum of 2 mM WTA

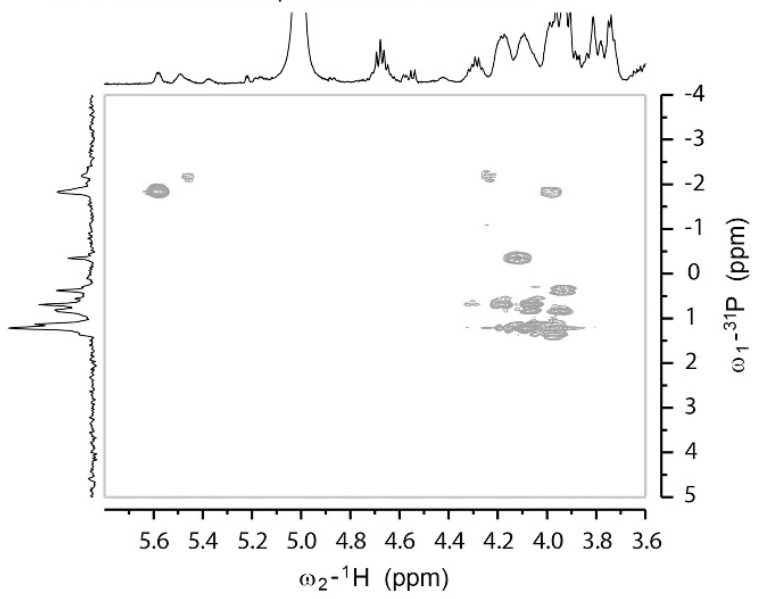

d ${ }_{1 \mathrm{H} / 31 \mathrm{P}} \mathrm{HMBC}$ NMR Spectrum of 2 mM WTA mixed with $3 \mathrm{mM}$ low-Mw BPEI

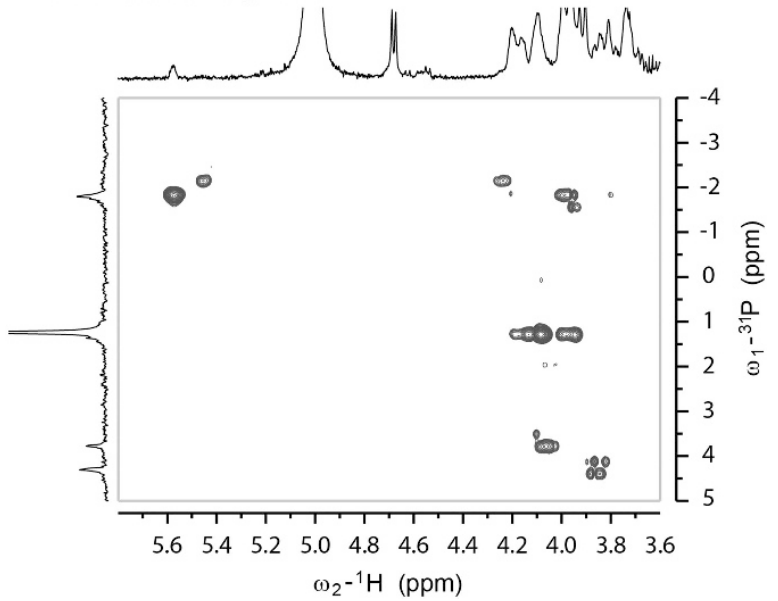

by MRSA and for the expression of $\beta$-lactam resistance in community-acquired strains. ${ }^{50}$ Thus, restoration of $\beta$-lactam activity in therapeutic clinical usage could be achieved with antibiotics or other compounds that target WTA synthesis or interrupt the ability of WTA to localize PBP2a in the proper configuration required for peptidoglycan crosslinking. If this perspective is true, there should be little or no benefit when BPEI and ampicillin are used to treat non-resistant $S$. aureus strains that do not express PBP2a. Supplementary Information (Supplementary Figure 6) show that the ampicillin MIC against methicillin-susceptible S. aureus ATCC 25923 was $50 \mathrm{ng} \mathrm{ml}^{-1}$. When combined with $2.65 \mu \mathrm{g} \mathrm{ml}^{-1}$ of BPEI, the ampicillin MIC of S. aureus ATCC 25923 was not reduced.

Rather than developing new inhibitors that require exhaustive clinical testing, we have discovered that some FDA-approved $\beta$-lactam antibiotics, such as ampicillin, can regain their efficacy against MRSA. The benefits to human health could be marked if the ampicillin+BPEI combination, used as a routine antibiotic therapy, can eliminate S. aureus infections, although simultaneously preventing the growth of ampicillin-resistant bacteria. Further experiments will be necessary to determine the extent of the BPEI-teichoic acid interaction and whether this interaction changes the structure of PBP2a. Importantly, the data for S. aureus ATCC 25923 provide a potential route to treat, and prevent, antibiotic-resistant infections. By using a combination of BPEI and ampicillin to treat a non-resistant $S$. aureus infection, the emergence of $\beta$-lactam resistant strains in vivo could be slowed. This benefit would not be possible with ampicillin alone. Formulations of an antibiotic with a compound that blocks the resistance pathway are a viable therapeutic strategy. For example, $\beta$-lactam antibiotics can be deactivated by bacteria that possess $\beta$-lactamases, a growing cause of resistance. ${ }^{51}$ Clavulanic acid is a $\beta$-lactamase inhibitor that restores $\beta$-lactam efficacy. ${ }^{51,52}$ The amoxicillin formulation is marketed as Augmentin (GlaxoSmithKline, Research Triangle Park, NC, USA) and now available in generic form. The success of $\beta$-lactam $+\beta$-lactamase inhibitor is an example that a combination therapy can be clinically and commercial viable. Although this approach effectively treats methicillin-susceptible S. aureus infections, MRSA bacteria will endure. ${ }^{53}$ Our approach has the potential of simultaneously eliminating methicillin-susceptible $S$. aureus and MRSA to limit tissue damage from toxins, decreasing morbidity and mortality. Patients will not have to endure multiple treatments with an array of antibiotics to clear the infection, thereby improving quality of life. ${ }^{54}$ Fewer medical complications and courses of treatment will result in better patient outcomes at a lower cost to patients and providers. ${ }^{54}$

Colonies of MRSA bacteria invade host tissue to release toxins that cause tissue injury, leading to significant patient morbidity. The patient suffers while numerous first- and second-line antibiotics are prescribed to no avail. This increases the threat of MRSA to public health. ${ }^{55}$ Timely MRSA diagnosis ${ }^{54}$ and delivering drugs of last resort are essential to prevent mortality. ${ }^{56}$ In 2011, MRSA infected 80500 people; nearly one in seven cases resulted in death $(11300 ; 14 \%) .{ }^{57}$ Several antibiotics of last resort (vancomycin, linezolid and

Figure $4{ }^{31} \mathrm{P}$ NMR spectra of WTA before and after the addition of low-MW BPEI (a, b) show significant changes in phosphate chemical shift caused by changes in the chemical environment. These changes are also manifested in the HMBC spectra (c, d). The P-31 signals near 4 p.p.m. are correlated with the proton signals of $\mathrm{N}$-acetylglucosamine sugar groups. However, clear identification of specific interactions is prevented by the heterogeneous nature of WTA functional groups, BPEI branching and WTA:BPEI-binding interactions. A full color version of this figure is available at The Journal of Antibiotics journal online. 
daptomycin) are effective at killing MRSA, and no MRSA strain is resistant to more than one of them. ${ }^{58,59}$ Although there has never been a $S$. aureus isolate resistant to all approved antibiotics, patients still die from MRSA infections. MRSA infections are deadly because drugs of last resort are given after morbidity from staphylococcal toxins, too late to prevent mortality. ${ }^{54,58}$ Vancomycin, a primary treatment option after MRSA diagnosis, presents additional barriers of high cost and toxicity. ${ }^{60-64} \mathrm{New}$ antibiotics, such as oxadiazoles, ${ }^{4}$ tedizolid ${ }^{5}$ and teixobactin, ${ }^{65}$ are awaiting FDA approval to meet the critical need for new treatments because $S$. aureus strains resistant to vancomycin and $\beta$-lactams have emerged. ${ }^{1,7,8}$ Although we envision improving human health with $\beta$-lactam plus polymer combinations, this represents a new pathway to develop other antibiotic treatments. Disabling PBP2a with cationic polymers enables advancement of antibiotic drug discovery by providing ways for other researchers to reinvigorate antibiotic development efforts that have stalled in the face of PBP2a.

\section{MATERIALS AND METHODS}

\section{Materials}

The bacteria used in this work were obtained from the American Type Culture Collection (MRSA) strain ATCC 700787, S. aureus ATCC 25923, E. coli ATCC 11775 and Bacillus subtilis (Ehrenberg) Cohn ATCC 23059). Chemicals from Sigma-Aldrich, Milwaukee, WI, USA (DMSO, ampicillin, vancomycin and novobiocin) were used as purchased.

\section{Preparation and characterization of cationic polymers}

Branched and linear polyethylenimine was obtained from Sigma-Aldrich as high-MW $(\sim 25 \mathrm{kDa})$ or low-MW $(\sim 0.5 \mathrm{kDa})$ polymers. The molecule has polycationic character from the protonation of its amine functional groups based on its protonation constant $\left(\mathrm{pK}_{\mathrm{a}}\right)$. Protonation constants for branched PEI molecules have been reported to be $\sim 4.5$ for primary amines, 6.7 for secondary amines and 11.6 for tertiary amines. ${ }^{66}$ Thus, at $\mathrm{pH}=7.2$, the BPEI has many positively charged primary amines to interact with teichoic acid.

\section{Growth inhibition assays}

Compounds were tested against MRSA strain ATCC 700787, which also exhibits reduced susceptibility to vancomycin. A stock culture was diluted in tryptic soy broth and delivered into pre-sterilized 96-well plates. Stock solutions of BPEI and antibiotics, prepared in DMSO, were added to each well of a 96-well plate (final DMSO concentration was 1\%), followed by inoculation with MRSA in tryptic soy broth. OD measurements were performed with a Tecan Infinite M200 plate reader (Tecan Inc, Männedorf, Switzerland) and an initial $\mathrm{OD}_{600}$ value recorded. The plates were incubated for $20 \mathrm{~h}$ in a humidified incubator at $37^{\circ} \mathrm{C}$. Plates were removed, orbitally shaken and a final $\mathrm{OD}_{600}$ value recorded. The final $\mathrm{OD}_{600}$ reading was subtracted from the initial $\mathrm{OD}_{600}$ reading to obtain the change in $\mathrm{OD}_{600}$ (recorded in the figures as $\Delta \mathrm{OD}_{600}$ ). Antimicrobial activity was determined by the change in OD. Duplicate measurements were performed and the average reported.

Using the average $\Delta \mathrm{OD}_{600}$ values, separate MIC values for BPEI and ampicillin were determined by the lowest concentration of each that inhibited growth. From this, FIC indices were calculated for all wells that showed inhibition. $^{22,23}$

\section{Cytotoxicity assay}

Mammalian cell cytotoxicity assays were performed on NIH/3T3 mouse fibroblast cells by adding 5000 cells per well into 96 -well plates. The cells were allowed to adhere overnight at $37^{\circ} \mathrm{C}$ in a humidified incubator $\left(5 \% \mathrm{CO}_{2}\right.$ atmosphere). Test compounds were diluted in DMSO and added to the wells so that the final concentration of DMSO per well did not exceed $1 \%$ by volume. The plates containing treated and control cells were incubated for $48 \mathrm{~h}$ and cell viability was determined by MTT assay. ${ }^{36}$ Duplicate measurements were performed and the average reported.

\section{Synthesis of the BPEI:dye conjugate}

Low-MW BPEI (Sigma-Aldrich) was added to Alexa Fluor 488 dye provided in the Alexa Fluor 488 Protein Labeling Kit (Thermo Fisher Scientific) at a ratio of $200 \mu \mathrm{l}$ BPEI ( $3 \mathrm{mg} \mathrm{ml}^{-1}$ stock in Milli-Q $\mathrm{H}_{2} \mathrm{O}$, Billerica, MA, USA) per tube of powdered dye. After allowing the dye and BPEI to form the conjugate over $1.5 \mathrm{~h}$ at $25^{\circ} \mathrm{C}$, the product was stored at $4{ }^{\circ} \mathrm{C}$ and used without further purification.

\section{Labeling MRSA cells with the BPEI:dye conjugate}

Cultures of E. coli ATCC 11775 and MRSA ATCC 700787 at mid-log growth phase were pelleted by centrifugation at $2000 \mathrm{~g}$ for $5 \mathrm{~min}$ at room temperature, and the growth media supernatant was removed. DAPI $(6 \mu \mathrm{M})$ in phosphatebuffered saline (1x, pH 7.2) was added to resuspend the cell pellet, which was allowed to incubate for $5 \mathrm{~min}$ at room temperature. The BPEI-dye conjugate was then added to a final concentration of $100 \mu \mathrm{g} \mathrm{ml}^{-1}$. Two control samples were prepared with either unconjugated BPEI or Alexa Fluor 488 alone and added to MRSA cells as described above. The stained bacterial cells were immediately fixed by resuspension with $4 \%$ paraformaldehyde followed by a 10 -fold dilution in 1x PBS. Cells were added to a microscope slide immediately before imaging.

\section{Confocal microscopy}

Paraformaldehyde-fixed cells were mounted in 1x PBS and imaged using a Leica SP8 LSCM (Leica, Buffalo Grove, IL, USA) with a $\times 63 / 1.4$ numerical aperture oil objective. A $405 \mathrm{~nm}$ GaN diode laser line was used to image DAPI, and a $488 \mathrm{~nm}$ argon laser line was used to observe Alexa Fluor 488 fluorescence. Single optical sections were acquired of cells that had adhered to the coverslip, for highest axial resolution, with a pixel resolution of $80 \times 80 \mathrm{~nm}$. Instrument settings were kept fixed for all imaging to allow for direct comparison of fluorescence intensity.

\section{Image processing}

To visualize the relative localization of fluorescence, independent of intensity, images were processed (ImageJ v1.49 m, National Institute of Mental Health, Bethesda, MD, USA) such that the total fluorescence intensity within each image was visible (Supplementary Figure 8, left column). To determine the relative fluorescence intensity between images, the fluorescence intensity of both the DAPI and the Alexa Fluor 488 were normalized to the respective intensities in the MRSA sample treated with BPEI conjugated with Alexa Fluor 488 (Supplementary Figure 8, right column).

\section{WTA purification for NMR studies}

The NMR experiments require 1-5 mg WTA isolated from 1 liter of bacterial cell culture. The poly(ribitol phosphate) WTA used in this work was isolated from B. subtilis W2367,68 (B. subtilis (Ehrenberg) Cohn ATCC 23059) rather than from MRSA, allowing high volumes (500-2000 ml) of culture to be processed with minimal risk to personnel. This also allows us to take the sample into the NMR facility room, which is not rated for BSL-2 work. In the rare instance of sample breakage, using WTA from B. subtilis W23 does not present a clean-up hazard nor require decontamination of the expensive NMR analysis probes, magnets or spectrometers.

B. subtilis W23 cells were grown in $\mathrm{LB}$ broth to an $\mathrm{OD}_{600}$ reading of $\sim 0.8$. After growth, the cells were collected by centrifugation and physically disrupted using an Avestin EmulsiFlex-C3 homogenizer (Ottawa, ON, Canada). The insoluble cell wall was collected, placed in boiling $6 \%(\mathrm{w} / \mathrm{v}) \mathrm{SDS}$, washed with sterile water and EDTA then placed in a $10 \%$ trichloroacetic acid treatment for $48 \mathrm{~h}$ at $4{ }^{\circ} \mathrm{C}$. After allowing the trichloroacetic acid to remove the bound WTA from the cell wall, the WTA was collected in the supernatant and placed into a $500 \mathrm{Da}$ MW cutoff dialysis membrane. Membrane dialysis was performed at $4{ }^{\circ}$ $\mathrm{C}$ in 1.5 liter of sterile water with continual water changes over a 24 -h period. The final $4 \mathrm{~h}$ of dialysis took place in a $1 \mathrm{kDa}$ MW cutoff membrane to assure sample purity. The sample was lyophilized and kept at $-20{ }^{\circ} \mathrm{C}$ until use. 


\section{NMR spectroscopy}

NMR samples were prepared in Eppendorf tubes by mixing teichoic acid with low-MW BPEI in water. The $\mathrm{pH}$ was measured with a microscopic $\mathrm{pH}$ probe and adjusted to 7.2 if necessary. A 3-mm NMR tube was filled with $160 \mu \mathrm{l}$ of a 2 mM sample of WTA/ $\mathrm{D}_{2} \mathrm{O}$ or a combination of $2 \mathrm{~mm}$ BPEI with $2 \mathrm{~mm}$ WTA in $\mathrm{D}_{2} \mathrm{O}$. NMR data collection use Agilent VNMRS-500 MHz NMR (Santa Clara, CA, USA) equipped with a pulsed field gradient probe tuned to the ${ }^{31} \mathrm{P}$ resonance frequency. Data acquisition and processing were performed using VNMRJ 2.2C software on a system running Red Hat Enterprise Linux.

\section{CONFLICT OF INTEREST}

The authors declare no conflict of interest.

\section{ACKNOWLEDGEMENTS}

This work is supported by the National Institutes of Health (CVR 1R01GM090064-01 and RHC 1R01AI085161), and the University of Oklahoma. DTG is grateful for partial support of this work by National Science Foundation (NSF; CBET 0967988) and Oklahoma Bioenergy Center (project no. 100020) grants. The confocal microscope used in the work was purchased with support from the NSF 1126578. We appreciate feedback from Dr Christopher Corbett, OU Director of Intellectual Property Management. CVR wishes to express our gratitude to Professor Jacob Schaefer (Washington University in St Louis) for insights regarding drug resistance in MRSA and Professor Cheryl Stevenson (Illinois State University, Normal, IL, USA) for helpful discussions.

1 Dhanalakshmi, T. A., Umapathy, B. L. \& Mohan, D. R. Prevalence of methicillin, vancomycin and multidrug resistance among Staphylococcus aureus. J. Clin. Diagn. Res. 6, 974-977 (2012).

2 Veni, E. J. K., Baliga, S., Shenoy, M. \& Gopalkrishna, B. K. Community-acquired methicillin resistant Staphylococcus aureus. Int. J. Curr. Res. Rev. 6, 1-10 (2014).

3 Keynan, Y. \& Rubinstein, E. Staphylococcus aureus bacteremia, risk factors, complications, and management. Crit. Care Clin. 29, 547-562 (2013).

4 O'Daniel, P. I. et al. Discovery of a new class of non- $\beta$-lactam inhibitors of penicillinbinding proteins with gram-positive antibacterial activity. J. Am. Chem. Soc. 136, 3664-3672 (2014).

5 Zhanel, G. G. et al. Tedizolid: a novel oxazolidinone with potent activity against multidrug-resistant gram-positive pathogens. Drugs 75, 253-270 (2015).

6 Ling, L. L. et al. A new antibiotic kills pathogens without detectable resistance. Nature 517, 455-459 (2015)

7 Appelbaum, P. C. The emergence of vancomycin-intermediate and vancomycinresistant Staphylococcus aureus. Clin. Microbiol. Infect. 12, 16-23 (2006).

8 Łęski, T. A. \& Tomasz, A. Role of penicillin-binding protein 2 (pbp2) in the antibiotic susceptibility and cell wall cross-linking of Staphylococcus aureus: evidence for the cooperative functioning of PBP2, PBP4, and PBP2a. J. Bacteriol. 187, 1815-1824 (2005).

9 Jiang, W. et al. Artesunate has its enhancement on antibacterial activity of $\beta$-lactams via increasing the antibiotic accumulation within methicillin-resistant Staphylococcus aureus (mrsa). J. Antibiot. 66, 339-345 (2013).

10 Chaudhari, K. \& Bajaj, H. K. The analysis of virulence factors and beta-lactamase production in clinical isolates of Staphylococcus aureus. Am. J. PharmTech Res. 4, 538-546 (2014).

11 Toussaint, K. A. \& Gallagher, J. C. B-lactam/ $\beta$-lactamase inhibitor combinations: from then to now. Ann. Pharmacother. 49, 86-98 (2015).

12 Hartman, B. J. \& Tomasz, A. Low-affinity penicillin-binding protein associated with beta-lactam resistance in Staphylococcus aureus. J. Bacteriol. 158, 513-516 (1984).

13 Fuda, C., Suvorov, M., Vakulenko, S. B. \& Mobashery, S. The basis for resistance to beta-lactam antibiotics by penicillin-binding protein 2a of methicillin-resistant Staphylococcus aureus. J. Biol. Chem. 279, 40802-40806 (2004).

14 Gardete, S. \& Tomasz, A. Mechanisms of vancomycin resistance in Staphylococcus aureus. J. Clin. Invest. 124, 2836-2840 (2014).

15 Farha, M. A. et al. Inhibition of WTA synthesis blocks the cooperative action of PBPs and sensitizes MRSA to $\beta$-lactams. ACS Chem. Biol. 8, 226-233 (2013).

16 Wang, H. et al. Discovery of wall teichoic acid inhibitors as potential anti-MRSA $\beta$ lactam combination agents. Chem. Biol. 20, 272-284 (2013).

17 Belcheva, A. \& Golemi-Kotra, D. A close-up view of the VraSR two-component system a mediator of Staphylococcus aureus response to cell wall damage. J. Biol. Chem. 283, 12354-12364 (2008).

18 Roemer, T., Schneider, T. \& Pinho, M. G. Auxiliary factors: a chink in the armor of MRSA resistance to beta-lactam antibiotics. Curr. Opin .Microbiol. 16, 538-548 (2013).

19 Moreillon, P. New and emerging treatment of Staphylococcus aureus infections in the hospital setting. Clin. Microbiol. Infect. 14, 32-41 (2008).
20 Brady, M. J., Lisay, C. M., Yurkovetskiy, A. V. \& Sawan, S. P. Persistent silver disinfectant for the environmental control of pathogenic bacteria. Am. J. Infect. Control. 31, 208-214 (2003).

21 Ubukata, K., Nonoguchi, R., Matsuhashi, M. \& Konno, M. Expression and inducibility in Staphylococcus aureus of the mecA gene, which encodes a methicillin-resistant S. aureus specific penicillin-binding protein. J. Bacteriol. 171, 2882-2885 (1989).

22 Odds, F. C. Synergy, antagonism, and what the chequerboard puts between them. J. Antimicrob. Chemo. 52, 1-1 (2003).

23 Johnson, M. D., MacDougall, C., Ostrosky-Zeichner, L., Perfect, J. R. \& Rex, J. H. Combination antifungal therapy. Antimicrob. Agents Chemother. 48, 693-715 (2004).

$24 \mathrm{Galm}$, U. et al. Antimicrobial and DNA gyrase-inhibitory activities of novel clorobiocin derivatives produced by mutasynthesis. Antimicrob. Agents Chemother. $\mathbf{4 8}$, 1307-1312 (2004).

25 Zhanel, G. G., Schweizer, F. \& Karlowsky, J. A. Oritavancin: Mechanism of action. Clin. Infect. Dis. 54, S214-S219 (2012).

26 Gibney, K. A. et al. Poly(ethylene imine)s as antimicrobial agents with selective activity. Macromol. Biosci. 12, 1279-1289 (2012).

27 Tang, G. P. et al. Low molecular weight polyethylenimines linked by beta-cyclodextrin for gene transfer into the nervous system. J. Gene Med. 8, 736-744 (2006).

28 Moghimi, S. M. et al. A two-stage poly(ethylenimine)-mediated cytotoxicity: Implications for gene transfer/therapy. Mol. Ther. 11, 990-995 (2005).

29 Zhou, J. Y., Yockman, J. W., Kim, S. W. \& Kern, S. E. Intracellular kinetics of non-viral gene delivery using polyethylenimine carriers. Pharm. Res. 24, 1079-1087 (2007).

30 Fuente, E. et al. Study of filler flocculation mechanisms and floc properties induced by polyethylenimine. Ind. Eng. Chem. Res. 44, 5616-5621 (2005).

31 Boussif, 0. et al. A versatile vector for gene and oligonucleotide transfer into cells in culture and in vivo: Polyethylenimine. Proc. Natl Acad. Sci 92, 7297-7301 (1995).

32 Taranejoo, S., Liu, J., Verma, P. \& Hourigan, K. A review of the developments of characteristics of PEI derivatives for gene delivery applications. J. Appl. Polym. Sci. 132, 42096 (2015)

33 Cubillos-Ruiz, J. R. et al. Polyethylenimine-based siRNA nanocomplexes reprogram tumor-associated dendritic cells via TLR5 to elicit therapeutic antitumor immunity. J Clin Invest. 119, 2231-2244 (2009).

34 Kunath, K. et al. Low-molecular-weight polyethylenimine as a nonviral vector for DNA delivery: comparison of physicochemical properties, transfection efficiency and in vivo distribution with high-molecular-weight polyethylenimine. J. Control. Release 89, 113-125 (2003).

35 Wagner, M., Rinkenauer, A. C., Schallon, A. \& Schubert, U. S. Opposites attract: Influence of the molar mass of branched poly(ethylene imine) on biophysical characteristics of siRNA-based polyplexes. RSC Adv. 3, 12774-12785 (2013).

36 Hansen, M. B., Nielsen, S. E. \& Berg, K. Re-examination and further development of a precise and rapid dye method for measuring cell growth/cell kill. J. Immunol. Methods 119, 203-210 (1989).

37 Khalil, H., Chen, T., Riffon, R., Wang, R. \& Wang, Z. Synergy between polyethylenimine and different families of antibiotics against a resistant clinical isolate of Pseudomonas aeruginosa. Antimicrob. Agents Chemother. 52, 1635-1641 (2008).

38 Helander, I. M., Alakomi, H.-L., Latva-Kala, K. \& Koski, P. Polyethyleneimine is an effective permeabilizer of gram-negative bacteria. Microbiology 143, 3193-3199 (1997).

39 Thomas, K. J. \& Rice, C. V. Revised model of calcium and magnesium binding to the bacterial cell wall. Biometals 27, 1361-1370 (2014).

40 Halye, J. L. \& Rice, C. V. Cadmium chelation by bacterial teichoic acid from solid-state nuclear magnetic resonance spectroscopy. Biomacromolecules 11, 333-340 (2010).

41 Wickham, J. R., Halye, J. L., Kashtanov, S., Khandogin, J. \& Rice, C. V. Revisiting magnesium chelation by teichoic acid with phosphorus solid-state NMR and theoretical calculations. J. Phys. Chem. B 113, 2177-2183 (2009).

42 da Silva, M. W. NMR methods for studying quadruplex nucleic acids. Methods 43 264-277 (2007).

43 Gotfredsen, C. H., Meissner, A., Duus, J. O. \& Sorensen, O. W. New methods for measuring H-1-P-31 coupling constants in nucleic acids. Magn. Reson. Chem. 38, 692-695 (2000).

44 Jennings, M. C., Buttaro, B. A., Minbiole, K. P. C. \& Wuest, W. M. Bioorganic investigation of multicationic antimicrobials to combat qac-resistant Staphylococcus aureus. ACS Infect. Dis. 1, 304-309 (2015).

45 Campbell, J. et al. Synthetic lethal compound combinations reveal a fundamental connection between wall teichoic acid and peptidoglycan biosyntheses in Staphylococcus aureus. ACS Chem. Biol. 6, 106-116 (2011).

46 D'Elia, M. A., Millar, K. E., Beveridge, T. J. \& Brown, E. D. Wall teichoic acid polymers are dispensable for cell viability in Bacillus subtilis. J. Bacteriol. 188, 8313-8316 (2006).

47 Bhavsar, A. P., Erdman, L. K., Schertzer, J. W. \& Brown, E. D. Teichoic acid is an essential polymer in Bacillus subtilis that is functionally distinct from teichuronic acid. J. Bacteriol. 186, 7865-7873 (2004).

48 Swoboda, J. G. et al. Discovery of a small molecule that blocks wall teichoic acid biosynthesis in Staphylococcus aureus. ACS Chem. Biol. 4, 875-883 (2009).

49 Pasquina, L. W., Santa Maria, J. P. \& Walker, S. Teichoic acid biosynthesis as an antibiotic target. Curr. Opin. Microbiol. 16, 531-537 (2013).

50 Atilano, M. L. et al. Teichoic acids are temporal and spatial regulators of peptidoglycan cross-linking in Staphylococcus aureus. Proc. Natl Acad. Sci. 107, 18991-18996 (2010).

51 Geddes, A. M., Klugman, K. P. \& Rolinson, G. N. Introduction: historical perspective and development of amoxicillin/clavulanate. Int. J. Antimicrob. Agents 30, S109-S112 (2007). 
52 Gill, E. E., Franco, O. L. \& Hancock, R. E. W. Antibiotic adjuvants: diverse strategies for controlling drug-resistant pathogens. Chem. Biol. Drug. Des. 85, 56-78 (2015).

53 Muller, S. et al. Poorly cross-linked peptidoglycan in MRSA due to mecA induction activates the inflammasome and exacerbates immunopathology. Cell Host Microbe. 18, 604-612 (2015)

54 Cosgrove, S. E. et al. The impact of methicillin-resistance in Staphylococcus aureus bacteremia on patient outcomes: mortality, length of stay, and hospital charges. Infect. Cont. Hosp. Epidemiol. 26, 166-174 (2005).

55 CDC. Antibiotic resistance threats in the United States 2013. Centers for Disease Control and Prevention, US Department of Health and Human Services (2013).

56 Kopp, B. J., Nix, D. E. \& Armstrong, E. P. Clinical and economic analysis of methicillinsusceptible and -resistant Staphylococcus aureus infections. Ann. Pharmacother. 38, 1377-1382 (2004)

57 Dantes, R. et al. National burden of invasive methicillin-resistant Staphylococcus aureus infections, United States, 2011. JAMA Intern. Med. 173, 1970-1978 (2013).

58 Pastagia, M., Kleinman, L. C., de la Cruz, E. G. L. \& Jenkins, S. G. Predicting risk for death from MRSA bacteremia. Emerg. Infect. Dis. 18, 1072-1080 (2012).

59 Mangili, A., Bica, I., Snydman, D. R. \& Hamera, D. H. Daptomycin-resistant, methicillin-resistant Staphylococcus aureus bacteremia. Clin. Infect. Dis. 40, 1058-1060 (2005)
60 Bosso, J. A. et al. Relationship between vancomycin trough concentrations and nephrotoxicity: a prospective multicenter trial. Antimicrob. Agents Chemother. 55, 5475-5479 (2011).

61 Koppula, S., Ruben, S., Bangash, F. \& Szerlip, H. M. Pitfalls in dosing vancomycin. Am. J. Med. Sci. 349, 137-139 (2015).

62 Bruniera, F. R. et al. The use of vancomycin with its therapeutic and adverse effects: a review. Eur. Rev. Med. Pharmacol. Sci. 19, 694-700 (2015).

63 Kurosu, M., Siricilla, S. \& Mitachi, K. Advances in MRSA drug discovery: where are we and where do we need to be? Expert Opin. Drug. Dis. 8, 1095-1116 (2013).

64 Wilke, M. H. Multiresistant bacteria and current therapy - the economical side of the story. Eur. J. Med. Res. 15, 571-576 (2010).

65 Ling, L. L. et al. A new antibiotic kills pathogens without detectable resistance. Nature 517, 455-459 (2015).

66 Demadis, K. D., Paspalaki, M. \& Theodorou, J. Controlled release of bis(phosphonate) pharmaceuticals from cationic biodegradable polymeric matrices. Ind. Eng. Chem. Res. 50, 5873-5876 (2011)

67 Swoboda, J. G., Campbell, J., Meredith, T. C. \& Walker, S. Wall teichoic acid function, biosynthesis, and inhibition. Chembiochem. 11, 35-45 (2010).

68 Brown, S., Meredith, T., Swoboda, J. \& Walker, S. Staphylococcus aureus and Bacillus subtilis W23 make polyribitol wall teichoic acids using different enzymatic pathways. Chem. Biol. 17, 1101-1110 (2010).

Supplementary Information accompanies the paper on The Journal of Antibiotics website (http://www.nature.com/ja) 